\title{
Convolution and correlation theorems for the windowed offset linear canonical transform*
}

\author{
Wen-Biao, Gao \\ School of Mathematics and Statistics \\ Beijing Institute of Technology \\ Beijing 102488, China
}

\author{
Bing-Zhao, Li \\ Beijing Key Laboratory on MCAACI \\ Beijing Institute of Technology \\ Beijing 102488, China \\ Email address:li_bingzhao@bit.edu.cn
}

\begin{abstract}
In this paper, some important properties of the windowed offset linear canonical transform (WOLCT) such as shift, modulation and orthogonality relation are introduced. Based on these properties we derive the convolution and correlation theorems for the WOLCT.
\end{abstract}

Index Terms-Windowed offset linear canonical transform, Convolution, Correlation

\section{INTRODUCTION}

The offset linear canonical transform (OLCT) [1], [2], [4], [5], [12], [13] is a generalized version of the linear canonical transform (LCT) with four parameters $(a, b, c, d)$ [6]-[11]. It is a six parameter $\left(a, b, c, d, u_{0}, w_{0}\right)$ class of linear integral transform. Because of the two extra parameters, time shifting $u_{0}$ and frequency modulation $w_{0}$, the OLCT are more general and flexible than the LCT. It has been widely applied in signal processing and optics [7]-[9], [11].

As a mathematical operation, the convolution provides some applications in pure and applied mathematics such as numerical linear algebra, numerical analysis, and signal processing [18], [19]. Correlation is similar to convolution and it is another useful operation in signal processing, optics and detection applications [20]-[22]. In some domains such as the LCT domain [3], [6], [13], Wignar-Ville transform domain [1], [8], [12] and the OLCT domain [1], [12], the convolution and correlation operations have been studied. They are the most fundamental and important theorems in these domains.

In [14], [15], [17], the window function based on linear canonical transform (WLCT) were presented. It is believed to be a new and important signal processing tool. They obtained some main properties such as covariance property, shift, modulation, orthogonality property and inversion formulas. The results of the WLCT have been well applied. Although the windowed offset linear canonical transform (WOLCT) [23] has been proposed, some properties of WOLCT has not been studied. The purpose of this paper is to study the WOLCT. Some properties of WOLCT are obtained and its

This work is supported by the National Natural Science Foundation of China (No. 61671063), and also by the Foundation for Innovative Research Groups of the National Natural Science Foundation of China (No. 61421001). convolution and correlation theorems are derived. The results are very important application in some fields such as digital signal and image processing.

In this paper we first review the OLCT. Next, we introduce the definition of the WOLCT, and obtain some important properties such as linearity, inversion formula and parity. Finally, we present the convolution and correlation theorems for the WOLCT.

\section{PRELIMINARY.}

Let us briefly review some basic properties.

The Lebesgue space $L^{2}(\mathbb{R})$ is defined as the space of all measurable functions on $\mathbb{R}$ such that

$$
\|f\|_{L^{2}(\mathbb{R})}=\left(\int_{\mathbb{R}}|f(t)|^{2} \mathrm{~d} t\right)^{\frac{1}{2}}<\infty
$$

Now we introduce an inner product of the functions $f, g$ defined on $L^{2}(\mathbb{R})$ is given by

$$
\langle f, g\rangle_{L^{2}(\mathbb{R})}=\int_{\mathbb{R}} f(t) \overline{g(t)} \mathrm{d} t .
$$

Definition 1. (OLCT) [1] Let $A=\left(a, b, c, d, u_{0}, w_{0}\right)$ be $a$ matrix parameter satisfying $a, b, c, d, u_{0}, w_{0} \in \mathbb{R}$, and ad$b c=1$. The OLCT of a signal $f(t) \in L^{2}(\mathbb{R})$ is defined by

$$
O_{A} f(u)=O_{A}[f(t)](u)= \begin{cases}\int_{-\infty}^{+\infty} f(t) K_{A}(t, u) \mathrm{d} t, & b \neq 0 \\ \sqrt{d} e^{i \frac{c d}{2}\left(u-u_{0}\right)^{2}+i u w_{0}} & \\ \times f\left(d\left(u-u_{0}\right)\right), & b=0\end{cases}
$$

where

$$
\begin{aligned}
K_{A}(t, u) & =\frac{1}{\sqrt{i 2 \pi b}} e^{i \frac{a}{2 b} t^{2}-i \frac{1}{b} t\left(u-u_{0}\right)-i \frac{1}{b} u\left(d u_{0}-b w_{0}\right)} \\
& \times e^{i \frac{d}{2 b}\left(u^{2}+u_{0}^{2}\right)}
\end{aligned}
$$

From definition 1 it can be seen that for case $b=0$ the OLCT is simply a time scaled version off multiplied by a linear chirp. Hence, without loss of generality, we assume $b \neq 0$. If $u_{0}=0$ and $w_{0}=0$, the OLCT reduces to the LCT [1], [3], [5], [7].

The inverse of an OLCT with parameters $A=$ $\left(a, b, c, d, u_{0}, w_{0}\right)$ is given by an OLCT with parameters 
$A^{-1}=\left(d,-b,-c, a, b w_{0}-d u_{0}, c u_{0}-a w_{0}\right)$. The exact inverse OLCT expression is [16]

$$
\begin{aligned}
f(t)=O_{A^{-1}}\left(O_{A} f(u)\right)(t) & =e^{i \frac{c d}{2} u_{0}^{2}-i a d u_{0} w_{0}+i \frac{a b}{2} w_{0}} \\
& \times \int_{-\infty}^{+\infty} O_{A} f(u) K_{A^{-1}}(u, t) \mathrm{d} u,
\end{aligned}
$$

Next, we introduce one of important properties for the OLCT, its generalized Parseval formula [4], [5], as follows:

$$
\left.\int_{\mathbb{R}} f(t) \overline{g(t)} \mathrm{d} t=\int_{\mathbb{R}} O_{A} f(u)\right) \overline{\left.O_{A} g(u)\right)} \mathrm{d} u
$$

III. THE WINDOWED OFFSET LINEAR CANONICAL TRANSFORM

Here we give definition of the WOLCT, then introduce some properties.

Definition 2. (WOLCT) [23] Let $\phi \in L^{2}(\mathbb{R}) \backslash\{0\}$ be a window function. The WOLCT of a signal $f \in L^{2}(\mathbb{R})$ with respect to $\phi$ is defined by

$$
V_{\phi}^{A} f(u, w)=\int_{\mathbb{R}} f(t) \overline{\phi(t-w)} K_{A}(t, u) \mathrm{d} t
$$

where $K_{A}(t, u)$ is given by (4).

For a fixed $w$, we have

$$
V_{\phi}^{A} f(u, w)=O_{A}[f(t) \overline{\phi(t-w)}](u)
$$

Using the inverse OLCT to (8), we have

$$
\begin{aligned}
f(t) \overline{\phi(t-w)} & =O_{A^{-1}}\left(V_{\phi}^{A} f(u, w)\right)(t) \\
& =e^{i \frac{c d}{2} u_{0}^{2}-i a d u_{0} w_{0}+i \frac{a b}{2} w_{0}} \\
& \times \int_{-\infty}^{+\infty} V_{\phi}^{A} f(u, w) K_{A^{-1}}(u, t) \mathrm{d} u,
\end{aligned}
$$

Some basic properties of the WOLCT are summarized in the following theorem.

Property 1 (Linearity). Let $\phi \in L^{2}(\mathbb{R}) \backslash\{0\}$ be a window function and $f, g \in L^{2}(\mathbb{R})$ the WOLCT is a linear operator, namely,

$$
\left[V_{\phi}^{A}(\lambda f+\mu g)\right](u, w)=\lambda V_{\phi}^{A} f(u, w)+\mu V_{\phi}^{A} g(u, w)
$$

for arbitrary constants $\lambda$ and $\mu$.

Proof. This follows directly from the linearity of the product and the integration involved in Definition 2.

Property 2 (Shift). Let $\phi \in L^{2}(\mathbb{R}) \backslash\{0\}$ be a window function and $f \in L^{2}(\mathbb{R})$. Then we have

$$
\begin{aligned}
V_{\phi}^{A}\left\{T_{t_{0}} f\right\}(u, w) & =V_{\phi}^{A}\{f\}\left(u-a t_{0}, w-t_{0}\right) \\
& \times e^{i a t_{0} w_{0}-i \frac{a c}{2} t_{0}^{2}+i c t_{0}\left(u-u_{0}\right)}
\end{aligned}
$$

where $T_{t_{0}} f(t)=f\left(t-t_{0}\right)$.
Proof. By Definition 2, we have

$$
V_{\phi}^{A}\left\{T_{t_{0}} f\right\}(u, w)=\int_{\mathbb{R}} f\left(t-t_{0}\right) \overline{\phi(t-w)} K_{A}(t, u) \mathrm{d} t
$$

By making the change of variable $x=t-t_{0}$ in the above expression, we obtain

$$
\begin{aligned}
V_{\phi}^{A}\left\{T_{t_{0}} f\right\}(u, w) & =\int_{\mathbb{R}} f(x) \overline{\phi\left(x-\left(w-t_{0}\right)\right)} \\
& \times K_{A}\left(x+t_{0}, u\right) \mathrm{d} x \\
& =\int_{\mathbb{R}} f(x) \overline{\phi\left(x-\left(w-t_{0}\right)\right)} Y \mathrm{~d} x \\
& =\int_{\mathbb{R}} f(x) \overline{\phi\left(x-\left(w-t_{0}\right)\right)} \\
& \times K_{A}\left(x, u-a t_{0}\right) \mathrm{d} x \\
& \times e^{i a t_{0} w_{0}-i \frac{a}{2 b} t_{0}^{2}(a d-1)+i \frac{1}{b} t_{0}\left(u-u_{0}\right)(a d-1)} \\
& =V_{\phi}^{A}\{f\}\left(u-a t_{0}, w-t_{0}\right) \\
& \times e^{i a t_{0} w_{0}-i \frac{a c}{2} t_{0}^{2}+i c t_{0}\left(u-u_{0}\right)}
\end{aligned}
$$

where $Y=\frac{1}{\sqrt{i 2 \pi b}} e^{i \frac{a}{2 b} x^{2}+i \frac{a}{2 b} t_{0}^{2}+i \frac{a}{b} x t_{0}-i \frac{1}{b} x\left(u-u_{0}\right)}$ $\times e^{-i \frac{1}{b} t_{0}\left(u-u_{0}\right)-i \frac{1}{b} u\left(d u_{0}-b w_{0}\right)+i \frac{d}{2 b}\left(u^{2}+u_{0}^{2}\right)}$.

Which completes the proof.

Property 3 (Modulation). Let $\phi \in L^{2}(\mathbb{R}) \backslash\{0\}$ be a window function and $f \in L^{2}(\mathbb{R})$. Then we have

$$
\begin{aligned}
V_{\phi}^{A}\left\{M_{s} f\right\}(u, w) & =V_{\phi}^{A}\{f\}(u-b s, w) \\
& \times e^{i b s w_{0}-i \frac{d b}{2} s^{2}+i d s\left(u-u_{0}\right)}
\end{aligned}
$$

where $M_{s} f(t)=f(t) e^{i s t}$.

Proof. From Definition 2, it follows that

$$
\begin{aligned}
V_{\phi}^{A}\left\{M_{s} f\right\}(u, w) & =\int_{\mathbb{R}} f(t) e^{i s t} \overline{\phi(t-w)} K_{A}(t, u) \mathrm{d} t \\
& =\int_{\mathbb{R}} f(t) \overline{\phi(t-w)} Y_{1} \mathrm{~d} t \\
& =\int_{\mathbb{R}} f(t) \overline{\phi(t-w)} K_{A}(t, u-b s) \mathrm{d} t \\
& \times e^{i b s w_{0}-i \frac{d b}{2} s^{2}+i d s\left(u-u_{0}\right)} \\
& =V_{\phi}^{A}\{f\}(u-b s, w) \\
& \times e^{i b s w_{0}-i \frac{d b}{2} s^{2}+i d s\left(u-u_{0}\right)}
\end{aligned}
$$

where $Y_{1}=\frac{1}{\sqrt{i 2 \pi b}} e^{i \frac{a}{2 b} t^{2}-i \frac{1}{b} t\left((u-b s)-u_{0}\right)-i \frac{1}{b} u\left(d u_{0}-b w_{0}\right)}$ $e^{i \frac{d}{2 b}\left(u^{2}+u_{0}^{2}\right)}$.

Which completes the proof.

Property 4 (Shift and modulation property). Let $\phi \in$ $L^{2}(\mathbb{R}) \backslash\{0\}$ be a window function and $f \in L^{2}(\mathbb{R})$. Then we have

$$
E V_{\phi}^{A}\left\{T_{t_{0}} M_{s} f\right\}(u, w)=V_{\phi}^{A}\{f\}\left(u-b s-a t_{0}, w-t_{0}\right)
$$


where $E=e^{i \frac{1}{b}\left(b s+a t_{0}\right)\left(d\left(u_{0}-u\right)+\frac{b}{2}\left(d s+c t_{0}\right)-b w_{0}\right)}$

$\times e^{i \frac{t_{0}}{2 b}\left(2 u-b s-2 u_{0}\right)}$.

Proof. The proof of Property 4 can be achieved by directly combining Properties 2 and Properties 3.

We will prove the inversion formula for the WOLCT by the connection between the OLCT and the WOLCT. From this theorem, we know that it is possible to restore the original signal $f$ perfectly using the inverse WOLCT as follows.

Property 5 (Inversion formula). Let $\phi, \psi \in L^{2}(\mathbb{R}) \backslash\{0\}$ be window function, $\langle\psi, \phi\rangle \neq 0$ and $f \in L^{2}(\mathbb{R})$. Then we get

$$
\begin{aligned}
f(t) & =\frac{1}{\langle\psi, \phi\rangle} e^{i \frac{c d}{2} u_{0}^{2}-i a d u_{0} w_{0}+i \frac{a b}{2} w_{0}} \int_{\mathbb{R}^{2}} V_{\phi}^{A} f(u, w) \\
& \times K_{A^{-1}}(u, t) \psi(t-w) \mathrm{d} u \mathrm{~d} w
\end{aligned}
$$

Proof. Multiplying both sides of (9) from the right by $\psi(t-$ $w)$ and integrating with respect to $\mathrm{dw}$ we get

$$
\begin{aligned}
\int_{\mathbb{R}} f(t) \overline{\phi(t-w)} \psi(t-w) \mathrm{d} w & =e^{i \frac{c d}{2} u_{0}^{2}-i a d u_{0} w_{0}+i \frac{a b}{2} w_{0}} \\
& \times \int_{\mathbb{R}^{2}} V_{\phi}^{A} f(u, w) K_{A^{-1}}(u, t) \\
& \times \psi(t-w) \mathrm{d} u \mathrm{~d} w
\end{aligned}
$$

Using (2), we have

$$
\begin{aligned}
f(t) & =\frac{1}{\langle\psi, \phi\rangle} e^{i \frac{c d}{2} u_{0}^{2}-i a d u_{0} w_{0}+i \frac{a b}{2} w_{0}} \int_{\mathbb{R}^{2}} V_{\phi}^{A} f(u, w) \\
& \times K_{A^{-1}}(u, t) \psi(t-w) \operatorname{dud} w
\end{aligned}
$$

which completes the proof.

$$
\begin{aligned}
& \text { If } \phi=\psi, \text { then } \\
& \qquad \begin{aligned}
f(t) & =\frac{1}{\|\phi\|^{2}} e^{i \frac{c d}{2} u_{0}^{2}-i a d u_{0} w_{0}+i \frac{a b}{2} w_{0}} \int_{\mathbb{R}^{2}} V_{\phi}^{A} f(u, w) \\
& \times K_{A^{-1}}(u, t) \phi(t-w) \mathrm{d} u \mathrm{~d} w
\end{aligned}
\end{aligned}
$$

Property 6 (Orthogonality relation for WOLCT). Let $\phi, \psi \in$ $L^{2}(\mathbb{R}) \backslash\{0\}$ be window function and $f, g \in L^{2}(\mathbb{R})$. Then we get

$$
\left\langle V_{\phi}^{A} f(u, w), V_{\psi}^{A} g(u, w)\right\rangle=\langle f, g\rangle\langle\psi, \phi\rangle
$$

Proof. From (6) and (8), we get

$$
\begin{aligned}
\left\langle V_{\phi}^{A} f(u, w), V_{\psi}^{A} g(u, w)\right\rangle & =\int_{\mathbb{R}} \int_{\mathbb{R}} V_{\phi}^{A} f(u, w) \\
& \times \overline{V_{\psi}^{A} g(u, w)} \mathrm{d} u \mathrm{~d} w \\
& =\int_{\mathbb{R}} \int_{\mathbb{R}} O_{A}\left[f(t) \overline{\phi\left(t^{\prime}\right)}\right](u) \\
& \times \overline{O_{A}\left[g(t) \overline{\psi\left(t^{\prime}\right)}\right](u)} \mathrm{d} u \mathrm{~d} w \\
& \left.=\int_{\mathbb{R}} \int_{\mathbb{R}} f(t) \overline{\phi\left(t^{\prime}\right)} \overline{(g(t)} \psi\left(t^{\prime}\right)\right) \mathrm{d} t \mathrm{~d} w \\
& =\int_{\mathbb{R}} f(t) \overline{g(t)}\left(\int_{\mathbb{R}} \psi\left(t^{\prime}\right) \overline{\phi\left(t^{\prime}\right)} \mathrm{d} w\right) \mathrm{d} t \\
& =\langle f, g\rangle\langle\psi, \phi\rangle
\end{aligned}
$$

where $t^{\prime}=t-w$ which completes the proof.

Based on the above theorem, we may conclude the following important consequences. (i) If $\phi=\psi$, then

$$
\left\langle V_{\phi}^{A} f(u, w), V_{\phi}^{A} g(u, w)\right\rangle=\langle f, g\rangle\|\phi\|^{2}
$$

(ii) If $f=g$, then

$$
\left\langle V_{\phi}^{A} f(u, w), V_{\psi}^{A} f(u, w)\right\rangle=\|f\|^{2}\langle\psi, \phi\rangle
$$

(iii) If $f=g$ and $\phi=\psi$, then

$$
\begin{array}{r}
\left\langle V_{\phi}^{A} f(u, w), V_{\phi}^{A} f(u, w)\right\rangle=\|f\|^{2}\|\phi\|^{2} \\
=\int_{\mathbb{R}} \int_{\mathbb{R}}\left|V_{\phi}^{A} f(u, w)\right|^{2} \mathrm{~d} u \mathrm{~d} w
\end{array}
$$

Property 7 (Parity). Let $\phi \in L^{2}(\mathbb{R}) \backslash\{0\}$ be a window function and $f \in L^{2}(\mathbb{R})$. Then we have

$V_{P \phi}^{A}\{P f\}(u, w)=V_{\phi}^{A}\{f\}\left(2 u_{0}-u,-w\right) e^{i 2 w_{0}\left(u-u_{0}\right)}$ where $\operatorname{Pf}(t)=f(-t)$.

Proof. From Definition 2, it follows that

$$
\begin{aligned}
V_{P \phi}^{A}\{P f\}(u, w) & =\int_{\mathbb{R}} f(-t) \overline{\phi(-(t-w))} K_{A}(t, u) \mathrm{d} t \\
& =\int_{\mathbb{R}} f(-t) \overline{\phi(-t-(-w))} K_{A}^{\prime} \mathrm{d} t \\
& \times e^{i \frac{1}{b}\left(2 u_{0}-u\right)\left(d u_{0}-b w_{0}\right)-i \frac{d}{2 b}\left(\left(2 u_{0}-u\right)^{2}+u_{0}^{2}\right)} \\
& \times e^{-i \frac{1}{b} u\left(d u_{0}-b w_{0}\right)+i \frac{d}{2 b}\left(u^{2}+u_{0}^{2}\right)} \\
& =V_{\phi}^{A}\{f\}\left(2 u_{0}-u,-w\right) e^{i 2 w_{0}\left(u-u_{0}\right)}
\end{aligned}
$$

where $K_{A}^{\prime}=\frac{1}{\sqrt{i 2 \pi b}} e^{i \frac{a}{2 b}(-t)^{2}-i \frac{1}{b}(-t)\left(\left(2 u_{0}-u\right)-u_{0}\right)}$ $\times e^{-i \frac{1}{b}\left(2 u_{0}-u\right)\left(d u_{0}-b w_{0}\right)+i \frac{d}{2 b}\left(\left(2 u_{0}-u\right)^{2}+u_{0}^{2}\right)}$.

Which completes the proof.

Property 8. Let $\phi \in L^{2}(\mathbb{R}) \backslash\{0\}$ be a window function and $f \in L^{2}(\mathbb{R})$. Then we have

$$
\begin{aligned}
V_{\bar{\phi}}^{A}\{\bar{f}\}(u, w) & =V_{f}^{A}\{\phi\}(u-a w,-w) \\
& \times e^{i c w\left(u_{0}-u\right)+i a w w_{0}-i \frac{a c}{2} w^{2}}
\end{aligned}
$$

Proof. From Definition 2, let $t-w=t_{1}$, then

$$
\begin{aligned}
V_{\bar{\phi}}^{A}\{\bar{f}\}(u, w) & =\int_{\mathbb{R}} \overline{f(t)} \phi(t-w) K_{A}(t, u) \mathrm{d} t \\
& =\int_{\mathbb{R}} \phi\left(t_{1}\right) \overline{f\left(t_{1}-(-w)\right)} \frac{1}{\sqrt{i 2 \pi b}} \\
& \times e^{i \frac{a}{2 b}\left(t_{1}+w\right)^{2}-i \frac{1}{b}\left(t_{1}+w\right)\left(\left(2 u_{0}-u\right)-u_{0}\right)} \\
& \times e^{-i \frac{1}{b} u\left(d u_{0}-b w_{0}\right)+i \frac{d}{2 b}\left(u^{2}+u_{0}^{2}\right)} \mathrm{d} t_{1} \\
& =\int_{\mathbb{R}} \phi\left(t_{1}\right) \overline{f\left(t_{1}-(-w)\right)} \frac{1}{\sqrt{i 2 \pi b}} \\
& \times e^{i \frac{a}{2 b} t_{1}^{2}-i \frac{1}{b} t_{1}\left((u-a w)-u_{0}\right)} \\
& \times e^{-i \frac{1}{b}(u-a w)\left(d u_{0}-b w_{0}\right)+i \frac{d}{2 b}\left((u-a w)^{2}+u_{0}^{2}\right)} \mathrm{d} t_{1} \\
& \times e^{i \frac{1}{b}(u-a w)\left(d u_{0}-b w_{0}\right)-i \frac{d}{2 b}\left((u-a w)^{2}+u_{0}^{2}\right)} \\
& \times e^{i \frac{a}{2 b} w^{2}-i \frac{1}{b} w\left(u-u_{0}\right)} \\
& \times e^{-i \frac{1}{b} u\left(d u_{0}-b w_{0}\right)+i \frac{d}{2 b}\left(u^{2}+u_{0}^{2}\right)} \\
& =V_{f}^{A}\{\phi\}(u-a w,-w) \\
& \times e^{i c w\left(u_{0}-u\right)+i a w w_{0}-i \frac{a c}{2} w^{2}}
\end{aligned}
$$

which completes the proof.

\section{CONVOLUTION AND CORRELATION THEOREMS FOR}

In this section, we derive convolution and correlation theorems for WOLCT. 
Definition 3 (OLCT Convolution). For any $f, g \in L^{2}(\mathbb{R})$, we define the convolution operation $\star$ for OLCT by

$$
(f \star g)(t)=\int_{\mathbb{R}} f(x) g(t-x) e^{-i \frac{a}{2 b} x(t-x)} \mathrm{d} x
$$

As a consequence of the above definition, we get the following important theorem:

Theorem 1 (WOLCT Convolution). Let $\phi \in L^{2}(\mathbb{R}) \backslash\{0\}$. Then, for every $f, g \in L^{2}(\mathbb{R})$, we have

$$
\begin{aligned}
V_{\phi \star \psi}^{A}(f \star g)(u, w) & =B \int_{\mathbb{R}} V_{\phi}^{A} f\left(m_{0}, m\right) V_{\psi}^{A} g\left(m_{1}, w-m\right) \\
& \times e^{i \frac{a}{2 b} m\left(\frac{d a}{2}-1\right)(m-w)} \mathrm{d} m
\end{aligned}
$$

where $m_{0}=u-\frac{a}{2}(w-m), m_{1}=u-\frac{a}{2} m, B=$ $\sqrt{i 2 \pi b} e^{i \frac{1}{b}\left(u-\frac{a}{2} w\right)\left(d u_{0}-b w_{0}\right)} e^{-i \frac{d a}{2 b} w\left(\frac{a}{4} w-u\right)-i \frac{a}{2 b}\left(u^{2}+u_{0}^{2}\right)}$.

Proof. Based on Definition 2 and Definition 3, we get

$$
\begin{aligned}
V_{\phi \star \psi}^{A}(f \star g)(u, w) & =\int_{\mathbb{R}}(f \star g)(t)(\bar{\phi} \star \bar{\psi})(t-w) K_{A}(t, u) \mathrm{d} t \\
& =\int_{\mathbb{R}} \int_{\mathbb{R}} f(x) g(t-x) e^{-i \frac{a}{2 b} x(t-x)} \mathrm{d} x \\
& \times \int_{\mathbb{R}} \bar{\phi}(r) \bar{\psi}(t-w-r) e^{-i \frac{a}{2 b} r(t-w-r)} \mathrm{d} r \\
& \times K_{A}(t, u) \mathrm{d} t
\end{aligned}
$$

Setting $x=x_{1}, t=x_{1}+x_{2}, r=x_{1}-m$, $y_{0}=i \frac{1}{b} u\left(d u_{0}-b w_{0}\right), y_{1}=i \frac{d}{2 b}\left(u^{2}+u_{0}^{2}\right)$, we get

$$
\begin{aligned}
V_{\phi \star \psi}^{A}(f \star g)(u, w) & =\int_{\mathbb{R}^{3}} f\left(x_{1}\right) g\left(x_{2}\right) \bar{\phi}\left(x_{1}-m\right) \\
& \times \bar{\psi}\left(x_{2}-(w-m)\right) e^{-i \frac{a}{b} x_{1} x_{2}} \\
& \times e^{+i \frac{a}{2 b} x_{1}(w-m)+i \frac{a}{2 b} m x_{2}-i \frac{a}{2 b} m(w-m)} \\
& \times \frac{1}{\sqrt{i 2 \pi b}} e^{i \frac{a}{2 b}\left(x_{1}+x_{2}\right)^{2}-i \frac{1}{b}\left(x_{1}+x_{2}\right)\left(u-u_{0}\right)} \\
& \times e^{-y_{0}+y_{1}} \mathrm{~d} x_{1} \mathrm{~d} x_{2} \mathrm{~d} m \\
& =\int_{\mathbb{R}^{3}} f\left(x_{1}\right) g\left(x_{2}\right) \bar{\phi}\left(x_{1}-m\right) \\
& \times \bar{\psi}\left(x_{2}-(w-m)\right) \frac{1}{\sqrt{i 2 \pi b}} \\
& \times e^{i \frac{a}{2 b} x_{1}^{2}-i \frac{1}{b} x_{1}\left(u-\frac{a}{2}(w-m)-u_{0}\right)+i \frac{a}{2 b} x_{2}^{2}} \\
& \times e^{-i \frac{1}{b} x_{2}\left(u-\frac{a m}{2}-u_{0}\right)-i \frac{a}{2 b} m(w-m)} \\
& \times e^{-y_{0}+y_{1}} \mathrm{~d} x_{1} \mathrm{~d} x_{2} \mathrm{~d} m \\
& =B \int_{\mathbb{R}} \int_{\mathbb{R}} f\left(x_{1}\right) \bar{\phi}\left(x_{1}-m\right) \\
& \times K_{A}\left(x_{1}, m_{0}\right) \mathrm{d} x_{1} \\
& \times \int_{\mathbb{R}} g\left(x_{2}\right) \bar{\psi}\left(x_{2}-(w-m)\right) \\
& \times K_{A}\left(x_{2}, m_{1}\right) \mathrm{d} x_{2} e^{i \frac{a}{2 b} m\left(\frac{d a}{2}-1\right)(m-w)} \mathrm{d} m \\
& =B \int_{\mathbb{R}} V_{\phi}^{A} f\left(m_{0}, m\right) V_{\psi}^{A} g\left(m_{1}, w-m\right) \\
& \times e^{i \frac{a}{2 b} m\left(\frac{d a}{2}-1\right)(m-w)} \mathrm{d} m \\
& =x^{2}(33)
\end{aligned}
$$

Definition 4 (OLCT Correlation). For any $f, g \in L^{2}(\mathbb{R})$, we define the correlation operation $\circ$ for OLCT by

$$
(f \circ g)(t)=\int_{\mathbb{R}} \overline{f(x)} g(x+t) e^{i \frac{a}{2 b} x(x+t)} \mathrm{d} x
$$

Next, we establish the correlation theorem for the WOLCT.

Theorem 2 (WOLCT Correlation). Let $\phi \in L^{2}(\mathbb{R}) \backslash\{0\}$. Then, for every $f, g \in L^{2}(\mathbb{R})$, we have

$$
\begin{aligned}
V_{\phi \circ \psi}^{A}(f \circ g)(u, w) & =B_{0} \int_{\mathbb{R}} V_{P \bar{\phi}}^{A}\{P \bar{f}\}\left(m_{2},-m\right) \\
& \times V_{\psi}^{A} g\left(m_{3}, m_{4}\right) e^{-i \frac{a}{2 b} m\left(\frac{d a}{2}-1\right) m_{4}} \mathrm{~d} m
\end{aligned}
$$

where $P \bar{f}(x)=\overline{f(-x)}, m_{2}=u-\frac{a}{2}(w+m), m_{3}=u+\frac{a}{2} m$, $B_{0}=\sqrt{i 2 \pi b} e^{i \frac{1}{b}\left(u-\frac{a}{2} w\right)\left(d u_{0}-b w_{0}\right)-i \frac{d a}{2 b} w\left(\frac{a}{4} w-u\right)-i \frac{a}{2 b}\left(u^{2}+u^{2}\right)}$ $m_{4}=w+m$

Proof. Based on Definition 3 and Definition 4, we get

$$
\begin{aligned}
V_{\phi \circ \psi}^{A}(f \circ g)(u, w) & =\int_{\mathbb{R}}(f \circ g)(t)(\bar{\phi} \circ \bar{\psi})(t-w) K_{A}(t, u) \mathrm{d} t \\
& =\int_{\mathbb{R}} \int_{\mathbb{R}} \overline{f(x)} g(x+t) e^{i \frac{a}{2 b} x(t+x)} \mathrm{d} x \\
& \times \int_{\mathbb{R}} \phi(r) \bar{\psi}(r+t-w) e^{i \frac{a}{2 b} r(r+t-w)} \mathrm{d} r \\
& \times K_{A}(t, u) \mathrm{d} t
\end{aligned}
$$


Setting $x=x_{1}, t=x_{2}-x_{1}, r=x_{1}-m$, we get

$$
\begin{aligned}
V_{\phi \circ \psi}^{A}(f \circ g)(u, w) & =\int_{\mathbb{R}^{3}} f\left(x_{1}\right) g\left(x_{2}\right) \phi\left(x_{1}-m\right) \\
& \times \bar{\psi}\left(x_{2}-m_{4}\right) \\
& \times e^{i \frac{a}{b} x_{1} x_{2}-i \frac{a}{2 b} x_{1} m_{4}-i \frac{a}{2 b} m x_{2}+i \frac{a}{2 b} m m_{4}} \\
& \times K_{A}\left(x_{2}-x_{1}, u\right) \mathrm{d} x_{1} \mathrm{~d} x_{2} \mathrm{~d} m \\
& =B_{0} \int_{\mathbb{R}} \int_{\mathbb{R}} \overline{f\left(x_{1}\right)} \phi\left(x_{1}-m\right) \\
& \times K_{A}\left(x_{1}, m_{2}\right) \mathrm{d} x_{1} \\
& \times \int_{\mathbb{R}} g\left(x_{2}\right) \bar{\psi}\left(x_{2}-m_{4}\right) K_{A}\left(x_{2}, m_{3}\right) \mathrm{d} x_{2} \\
& \times e^{-i \frac{a}{2 b} m\left(\frac{d a}{2}-1\right)(m+w)} \mathrm{d} m \\
& =B_{0} \int_{\mathbb{R}} V_{P \bar{\phi}}^{A}\{P \bar{f}\}\left(m_{2},-m\right) \\
& \times V_{\psi}^{A} g\left(m_{3}, m_{4}\right) e^{-i \frac{a}{2 b} m\left(\frac{d a}{2}-1\right) m_{4}} \mathrm{~d} m
\end{aligned}
$$

where $K_{A}\left(x_{1}, m_{2}\right)=\frac{1}{\sqrt{i 2 \pi b}} e^{i \frac{a}{2 b}\left(-x_{1}\right)^{2}-i \frac{1}{b}\left(-x_{1}\right)}$

$$
\times e^{u-\frac{a}{2}(w+m)-u_{0}}
$$$$
\times e^{-i \frac{1}{b}\left(u-\frac{a}{2}(w+m)\right)\left(d u_{0}-b w_{0}\right)+i \frac{d}{2 b}\left(\left(u-\frac{a}{2}(w+m)\right)^{2}+u_{0}^{2}\right)},
$$$$
K_{A}\left(x_{2}, m_{3}\right)=\frac{1}{\sqrt{i 2 \pi b}} e^{i \frac{a}{2 b} x_{2}^{2}-i \frac{1}{b} x_{2}\left(u+\frac{a}{2} m-u_{0}\right)}
$$$$
\times e^{-i \frac{1}{b}\left(u+\frac{a}{2} m\right)\left(d u_{0}-b w_{0}\right)+i \frac{d}{2 b}\left(\left(u+\frac{a}{2} m\right)^{2}+u_{0}^{2}\right)} .
$$

Corollary 3. If the parameter of WOLCT changes to $\left(a, b, c, d, u_{0}, w_{0}\right)=(0,1,-1,0,0,0)$, then the Theorem 2 reduces to correlation theorem as follows:

$$
\begin{aligned}
V_{\phi \circ \psi}^{A}(f \circ g)(u, w) & =\sqrt{i 2 \pi b} e^{-i u w_{0}} \int_{\mathbb{R}} V_{P \bar{\phi}}^{A}\{P \bar{f}\}(u,-m) \\
& \times V_{\psi}^{A} g(u, w+m) \mathrm{d} m
\end{aligned}
$$

\section{CONCLusion}

In this paper, based on the association between the window function and the OLCT, we have studied the WOLCT. We first study some properties of the WOLCT, such as shift, modulation and orthogonality relation. Then, the convolution and correlation theorems for the WOLCT are shown. In our future works, the applications of the convolution and correlation theorems in signal and image processing will be investigated, and sampling theorem for the WOLCT and its applications will be studied.

\section{REFERENCES}

[1] D. Urynbassarova, B. Z. Li, and R. Tao, The Wigner-Ville distribution in the linear canonical transform domain, IAENG International Journal of Applied Mathematics. 46 (no. 4(December)) (2016) 559-563.
[2] S. Abe, J. T. Sheridan, Optical operations on wave functions as the Abelian Subgroups of the special affine Fourier transformation, Opt. Lett 19 (no. 22)(1994) 1801-1803.

[3] N. Goel, and K. Singh, A modified convolution and product theorem for the linear canonical transform derived by representation transformation in quantum mechanics. International Journal of Applied Mathematics and Computer Science, 2013, 23(3): 685-695.

[4] X. Zhi, D. Wei, and W. Zhang, A generalized convolution theorem for the special affine Fourier transform and its application to filtering, Optik 127 (5) (2016) 2613-2616.

[5] A. Stern, Sampling of compact signals in offset linear canonical transform domains, Signal Image Video Process 1 (4) (2007) 359-367.

[6] Q. Feng, and B. Z. Li, Convolution and correlation theorems for the two-dimensional linear canonical transform and its applications. IET Signal Processing, 2016, 10(2): 125-132.

[7] T. Z. Xu, and B. Z. Li, Linear Canonical Transform and Its Application, Beijing, Science Press, 2013.

[8] M. Bahri, and R. Ashino, Convolution and correlation theorems for Wigner-Ville distribution associated with linear canonical transform, 12th International Conference on Information Technology. (2015).

[9] R. Tao, B. Z. Li, Y. Wang, and G. K. Aggrey, On sampling of bandlimited signals associated with the linear canonical transform, IEEE Transactions on Signal Process 56 (11) (2008) 5454-5464.

[10] R. F. Bai, B. Z. Li, and Q. Y. Cheng, Wigner-Ville distribution associated with the linear canonical transform, Journal of Applied Mathematics. 2012 (2012) 1-14.

[11] L. Xiao, and W. Sun, Sampling theorems for signals periodic in the linear canonical transform domain, Optics Communications 290 (2013) 14-18.

[12] D. Urynbassarova, A. Urynbassarova, and E. Al-Hussam, The WignerVille distribution based on the offset linear canonical transform domain, 2nd International Conference on Modelling, Simulation and Applied Mathematics, (March 2017) (2017).

[13] J. Shi, X. Liu, and N. Zhang. Generalized convolution and product theorems associated with linear canonical transform. Signal, Image and Video Processing, 2014, 8(5): 967-974.

[14] K. I. Kou and R. H. Xu, Windowed linear canonical transform and its applications, Signal Processing, vol. 92, no. 1, pp. 179188, 2012.

[15] K. I. Kou, R. H. Xu and Y. H. Zhang, Paley-Wiener theorems and uncertainty principles for the windowed linear canonical transform, Mathenatical Methods in the Applied Science. 35 (2012) 2122-2132.

[16] Q. Xiang, and K. Y. Qin, Convolution, correlation, and sampling theorems for the offset linear canonical transform, Signal Image Video Process. 2014 (no.8) (2012) 433-442.

[17] M. Bahri, and R. Ashino, Some properties of windowed linear canonical transform and its logarithmic uncertainty principle. International Journal of Wavelets, Multiresolution and Information Processing, 2016, 14(03).

[18] J. Ebling, and G. Scheuermann, Clifford Fourier transform on vector fields, IEEE Transactions on Visualization and Computer Graphics, 2005, 11, (4), pp. 469-479.

[19] E. Hitzer,and B. Mawardi, Clifford Fourier transform on multivector 
fields and uncertainty principles for dimensions, Advances in Applied Clifford Algebras, 2008, 18, (3-4), pp. 715-736.

[20] O. Akay, and G. F. Boudreaux-Bartels, Fractional convolution and correlation via operator methods and application to detection of linear FM signals, IEEE Transactions on Signal Processing, 2001, 49, (5), pp. 979-993.

[21] S. C. Pei, and J. J. Ding, Relations between fractional operations and time-frequency distributions and their applications, IEEE Transactions on Signal Processing, 2001, 49, (8), pp. 1638-1655.

[22] Z. C. Zhang, Sampling theorem for the short-time linear canonical transform and its applications. Signal Processing, 2015, 113: 138-146.

[23] H. Y. Huo, W. C. Sun, L. Xiao, Uncertainty principles associated with the offset linear canonical transform. Mathematical Methods in the Applied Sciences. 2019; 42(2): 466-474. 